\title{
variations de volume des sols argileux lors de cycles de drainage-humidification
}

\author{
volumetric variations of clayey soils \\ during drying-wetting cycles
}

\author{
Jean BIAREZ \\ Professeur" \\ Jean-Marie FLEUREAU \\ Chef de travaux* \\ Moulay-Idriss ZERHOUNI \\ Chercheur* \\ Budi Susilo SOEPANDJI \\ Chercheur ${ }^{*}$
}

\section{Résumé}

Un travail expérimental a été effectué afin de préciser le comportement volumique de trois sols argileux (limon, marne, kaolinite) soumis à un cycle de drainage-humidification. La mesure simultanée des teneurs en eau et des variations de volume et leur représentation dans un plan $\left(e-u_{c}-w-S_{,}\right)$a permis de définir à chaque instant l'état complet du matériau et notamment son degré de saturation, pour différents modes de préparation.

D'autre part, la comparaison entre chemins de drainage-humidification et chemins de compression-décompression œdométriques et isotropes a mis en évidence une équivalence du rôle des contraintes capillaire et mécanique.

\footnotetext{
Abstract

An experimental work was carried out to assess the volumetric behaviour of three clayey soils (loam, marl, kaolinite) under the effect of a drying-wetting cyle. The simultaneous measurement of water contents and volume changes and their representation in a (e- $u_{c}-w-S$ ) plane gives, for each value of $u_{c i}$ the complete state of the material and its saturation domain, for different techniques of preparation.

Moreover, the comparison between drying-wetting paths and œdometric or isotropic compression-decompression paths showed an equivalence between the effects of the capillary and mechanical stresses.
}

\footnotetext{
* École centrale de Paris, Laboratoire de Mécanique des Sols-Structures, UA C.N.R.S. 850, 92295 Chatenay-Malabry. France.

- Adresse actuelle : Université d'Indonésie, Département de Génie Civil, Jakarta, Indonésie.
} 


\section{INTRODUCTION}

De très nombreux problèmes géotechniques font intervenir les variations de volume du sol sous l'action de contraintes d'origine capillaire : l'un des principaux est le retrait-gonflement des sols situés au-dessus de la nappe phréatique, mais aussi la géotechnique routière, le comportement des matériaux compactés, les phénomènes d'infiltration et d'évaporation, etc.

En l'absence de contrainte mécanique, le volume du sol peut varier si l'on soumet l'eau contenue dans l'échantillon à un état de tension (l'air restant à la pression atmosphérique). Plus généralement, c'est la différence entre les pressions interstitielles de l'air et de l'eau - ou pression capillaire - qui est le paramètre déterminant des mouvements des fluides dans le milieu poreux :

$$
\mathrm{u}_{\mathrm{c}}=\mathrm{u}_{\mathrm{a}}-\mathrm{u}_{\mathrm{w}}{ }^{*}
$$

A l'échelle d'un pore, cette différence de pression se traduit par l'existence d'un ménisque entre l'air et l'eau. Elle est caractérisée par la tension superficielle de l'eau et la courbure de l'interface (Loi de LAPLACE). La notion de pression capillaire est une notion globale, définie dans un domaine de dimensions très supérieures aux dimensions moléculaires, qui intègre à la fois les propriétés capillaires du milieu - caractérisées par le "rayon" des pores - et les propriétés d'adsorption de l'argile par l'intermédiaire de l'angle de raccordement solide-liquide-air. En fait, compte tenu de l'existence, au voisinage des particules d'argile d'une couche diffuse d'eau adsorbée, l'angle de raccordement doit être lui-même considéré comme un paramètre macroscopique déduit, par exemple, d'un travail ou d'une chaleur de mouillage (WIGNYODARSONO, 1985).

Lorsque les contraintes externes sont nulles, la pression capillaire est identique à la succion (s) des agronomes et l'on définit le $\mathrm{pF}$ :

$$
\mathrm{pF}=\log (\mathrm{s})=\log \left(\mathrm{u}_{\mathrm{c}}\right),
$$

$s$ et $u_{c}$ étant exprimées en centimètres de colonne d'eau. Néanmoins, la pression capillaire sera utilisée de préférence à la succion dans la mesure où sa définition peut être généralisée à n'importe quel état de contrainte mécanique, contrairement à la succion.

De nombreuses études expérimentales ont été effectuées afin de relier l'état de compacité ou d'humidité d'un sol et la pression capillaire en l'absence de contrainte mécanique (CRONEY, 1960; COLEMAN. 1961: VERBRUGGE, 1974: TESSIER, 1979: DANIEL, 1983). Les essais ont mis en évidence, de façon un peu dispersée, les paramètres prépondérants de comportement volumique du sol. Par ailleurs, certains chercheurs (JENNINGS, 1960; BLIGHT, 1966), en comparant les résultats d'essais de consolidation mécanique et de drainage sur échantillons saturés, ont

- Par convention, les pressions et les contraintes seront toujours prises par référence à la pression atmosphérique. montré l'équivalence entre l'action d'une contrainte mécanique et celle de la pression capillaire. Une tentative de synthèse de ces recherches a été faite par BISHOP (1963) qui a proposé une expression des contraintes effectives pour les matériaux partiellement saturés :

$$
\sigma^{\prime}=\left(\sigma-u_{\partial}\right)+x_{c}
$$

Le problème essentiel posé par cette relation réside dans la détermination expérimentale du paramètre $\chi$. Les études de BLIGHT (1967), BARDEN (1969) ont montré que le paramètre $x$ dépendait du chemin suivi et ne permettait pas de rendre compte de façon satisfaisante du comportement du matériau, notamment du point de vue des variations de volume et lors du relâchement de la pression capillaire jusqu'à zéro. Ces résultats ont conduit MATYAS (1968), BARDEN (1969), FREDLUND (1976, 1977), à remplacer le concept de contraintes effectives par celui de variables indépendantes, $\sigma-u_{\mathrm{a}}$ et $\mathrm{u}_{c}$, qui déterminent la résistance au cisaillement et les variations de volume du sol. Dans ces conditions le critère de rupture de MOHRCOULOMB s'écrit :

$$
\left(\tau=c^{t}+\left(\sigma-u_{a}\right) \operatorname{tg} \varnothing^{\prime}+\left(u_{c}\right) \operatorname{tg} \varnothing_{b},\right.
$$

$\varnothing^{\prime}$ et $\varnothing_{b}$ étant deux constantes, ce qui représente l'équation d'un plan dans les axes $\tau$, $a-u_{\text {, et }} u_{\text {c }}$ (GULHATI, 1981; HO, 1982). En fait, différents résultats expérimentaux (VERBRUGGE, 1978; LLO. RET, 1985) ont montré que la surface de rupture n'était pas un plan en dehors du domaine saturé et que le paramètre $\varnothing_{b}$ pouvait, comme $\chi$, dépendre du chemin suivi. Malheureusement, le relativement petit nombre d'expériences disponibles ne permet pas de conclure de façon définitive sur la validité de ces deux théories (ZERHOUNI, 1985).

L'étude présentée dans cet article constitue une pre. mière approche dans la recherche de la modélisation du comportement mécanique des sols argileux soumis à des états de contraintes capillaires. Celle-ci est abordée ici exclusivement sous l'aspect des variations de volume. Après avoir examiné le comportement volumique de trois matériaux (kaolinite, limon, marne) lors d'un cycle de drainage-humidification dans le domaine saturé, et notamment la comparaison avec les résultats des essais de compression-décompression cedométriques et isotropes, on étudiera l'influence de l'état initial et du remaniement sur les caractéristiques saturées et non saturées des échantillons.

\section{MÉTHODE EXPÉRIMENTALE ET MATÉRIAUX ÉTUDIÉS}

\subsection{Appareillages et mesures effectuées}

Comme il a été indiqué précédemment, la pression capillaire est la différence des pressions des phases air et eau :

$$
\mathrm{u}_{\mathrm{c}}=\mathrm{u}_{\mathrm{a}}-\mathrm{u}_{\mathrm{w}}
$$


Par conséquent, celle-ci peut être obtenue indifféremment en diminuant la pression de l'eau, $u_{w}$, ou en augmentant la pression de l'air, $\mathrm{u}_{\mathrm{a}}$. La première technique est employée dans les plaques tensiométriques dans lesquelles l'eau est mise en tension par rapport à l'air, à la pression atmosphérique. Elle est limitée en pratique à quelques dizaines de $\mathrm{kPa}$ (désaération de l'eau, etc.). La seconde méthode est celle des cellules à pression : l'eau étant à la pression atmosphérique, on augmente la pression de l'air jusqu'à la valeur de $\mathrm{u}_{c}$ choisie, limitée d'une part par la résistance mécanique de l'enceinte, d'autre part par la pression d'entrée d'air de la membrane semi-perméable. Cette membrane, commune aux deux méthodes précédentes, permet de fixer de façon indépendante les pressions des phases air et eau. La taille des pores et la nature du matériau du filtre réalisent cette séparation. En pratique, les plaques tensiométriques munies de membranes en verre fritté ont été utilisées jusqu'à une pression de $20 \mathrm{kPa}$. Les cellules à pression, équipées de diverses membranes cellulosiques (OXOID NUFLOW, VISKING), ont permis d'atteindre $1500 \mathrm{kPa}$. Dans tous les appareils, lobtention d'un bon contact entre les membranes et les échantillons - notamment préconsolidés - a été assurée par une mince couche de pâte de kaolin (TESSIER, 1977). Pour les appareils à pression, l'évacuation de l'air dissout dans l'eau, diffusant à travers la membrane semi-perméable, a été réalisée par des systèmes de pompage et de purge.

Pour les valeurs de pression capillaire plus élevées (2 à $1000 \mathrm{MPa}$ ), un dispositif tout à fait différent est utilisé, qui fait appel à des solutions salines saturées. En effet, la pression de vapeur au voisinage d'un ménisque est directement reliée à la courbure de l'interface (Loi de KELVIN) :

$$
k T \operatorname{Ln} P^{\prime} / P=2 A \cdot M / R \rho
$$

(PADDAY. 1969) dans laquelle M représente la masse moléculaire du liquide, $\rho$ sa densité, A sa tension superficielle, $\mathrm{k}$ la constante de BOLTZMANN, $\mathrm{T}$ la température, et $\mathrm{P}^{\prime}$ et $\mathrm{P}$ les pressions de vapeur du liquide respectivement au-dessus d'une surface plane et au-dessus d'une surface sphérique de rayon $\mathrm{R}$. Il en résulte donc qu'un équilibre s'établit entre la pression capillaire et la pression de vapeur du liquide $\mathrm{P}_{\mathrm{y}}$, ou le degré d'humidité relative $\mathrm{P}_{\mathrm{v}} / \mathrm{P}_{\mathrm{vs}}$ (Loi de LAPLACE). Le contrôle de l'humidité relative de l'air est assuré par la compétition entre la tendance de l'eau à saturer l'atmosphère de l'enceinte fermée (dessicateur) dans laquelle est placée la solution saline et celle des sels à s'hydrater. L'échantillon placé dans l'atmosphère du dessicateur parvient, en général après plusieurs semaines, à un équilibre de pression capillaire, qui dépend de la nature de la solution saline saturée (Tableau I).

\begin{tabular}{|l|r|}
\hline \multicolumn{1}{|c|}{ Solution saturée } & Pression capillaire \\
\hline $\mathrm{CuSO}_{4}, 5 \mathrm{H}_{2} \mathrm{O}$ & $1,6 \mathrm{MPa}$ \\
$\mathrm{ZnSO}_{4}, 7 \mathrm{H}_{2} \mathrm{O}$ & $12,6 \mathrm{MPa}$ \\
$\mathrm{KCl}$ & $22,4 \mathrm{MPa}$ \\
$\mathrm{NaCl}$ & $33,1 \mathrm{MPa}$ \\
$\mathrm{NaNO}_{2}$ & $60,3 \mathrm{MPa}$ \\
$\mathrm{CaCl}_{2}, 6 \mathrm{H}_{2} \mathrm{O}$ & $158,5 \mathrm{MPa}$ \\
$\mathrm{H}_{2} \mathrm{SO}_{4}(\mathrm{~d}=1,81)$ & $398,1 \mathrm{MPa}$ \\
\hline
\end{tabular}

Tableau I. - Pressions capillaires mesurées correspondant aux solutions salines saturées utilisées (à température ambiante).

Table I. - Capillary pressures for the saturated solutions of salts used.

Une fois cet équilibre atteint pour une valeur de pres. sion capillaire donnée, on mesure la teneur en eau de l'échantillon, et son volume total par immersion dans du Kerdane, après une imbibition préalable d'une

\begin{tabular}{|c|c|c|c|}
\hline & Limon & Marne & Kaolinite \\
\hline Minéralogie & & $\begin{array}{l}\text { Dolomite + } \\
\text { Calcite } 48-64 \% \\
\text { Anhydrite + } \\
\text { Gypse } 13-22 \% \\
\text { Argiles } 16-21 \%\end{array}$ & Kaolinite $100 \%$ \\
\hline $\begin{array}{l}\text { Granulométrie } \\
\text { inf. } 80 \mu \\
\text { inf. } 2 \mu \\
D_{60} / D_{10}\end{array}$ & $\begin{array}{l}98 \% \\
13 \% \\
27\end{array}$ & $\begin{array}{c}90-95 \% \\
5-10 \% \\
4\end{array}$ & $\begin{array}{c}100 \% \\
75 \% \\
11\end{array}$ \\
\hline $\begin{array}{l}\text { Lim. d'ATTERBERG } \\
w_{L} \\
w_{P} \\
\text { IP }\end{array}$ & $\begin{array}{r}27 \% \\
20 \% \\
7 \%\end{array}$ & $\begin{array}{c}34-38 \% \\
21-24 \% \\
13 \%\end{array}$ & $\begin{array}{l}61 \% \\
30 \% \\
31 \%\end{array}$ \\
\hline $\begin{array}{c}\text { Dens. des grains } \\
\qquad \gamma_{s} / \gamma_{w}\end{array}$ & 2,70 & 2,75 & 2,65 \\
\hline
\end{tabular}

Tableau II. - Caractéristiques des matériaux étudiés. Table II. - Characteristics of the soils studied. 
heure. La différence entre le poids de l'échantillon imbibé de Kerdane et son poids immergé permet d'obtenir le volume total, d'où l'on déduit l'indice des vides et le degré de saturation.

\subsection{Caractéristiques des échantillons}

Trois matériaux ont été utilisés (Tableau II) :

- une kaolinite artificielle pratiquement pure;

- un limon naturel moyennement plastique;

- une marne fortement cimentée à l'état naturel, utilisée ici sous forme remaniée et non remaniée.

\subsection{Préparation des échantillons remaniés}

Les échantillons remaniés sont préparés à partir d'une pâte saturée, obtenue par mélange de poudre et d'eau à une teneur en eau égale à 1,2 ou 1,5 fois la limite de liquidité. Dans le cas de la kaolinite, ceux-ci ont été ensuite consolidés par drainage partiel (dans une cellule à pression jusqu'à $u_{c}=1,5 \mathrm{MPa}$ ) ou totale (dans une étuve : $u_{c}=1000 \mathrm{MPa}$, par convention), afin d'étudier le rôle de l'état initial de l'argile. Les chemins suivis pour la préparation des différentes séries d'essais (K1 à K6) sont représentés sur la figure 6.

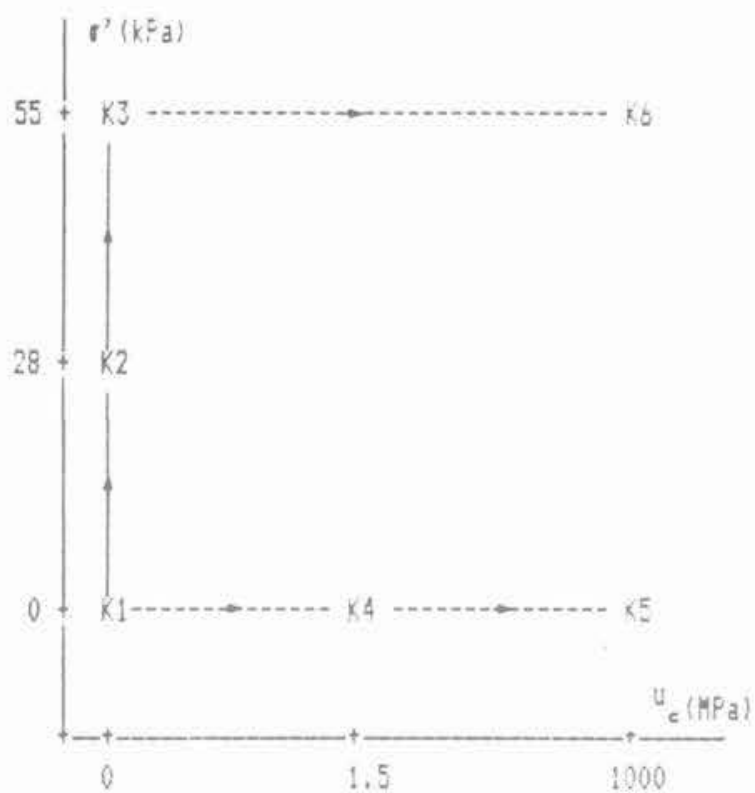

Fig. 6. - Représentation schématique des états de contrainte (mécanique et capillaire) initiaux des échantillons de kaolinite.

Fig. 6. - Initial (mechanical and capillary) stress state of the kaolonite samples.

\section{RÉSULTATS ET DISCUSSION}

3.1. Comparaison des essais de drainagehumidification et de compression œdométrique et isotrope

Les figures 1 et 2 présentent sur le même graphique, d'une part les courbes de drainage-humidification tra- cées en fonction du logarithme de la pression capillaire $u_{c}$, d'autre part les courbes de compressiondécompression œdométriques et isotropes sur échantillons saturés de kaolinite et de marne, en fonction du logarithme de la pression moyenne $p$ :

$$
\mathrm{p}=\left(\sigma_{1}^{\prime}+2 \sigma_{3}^{\prime}\right) / 3
$$

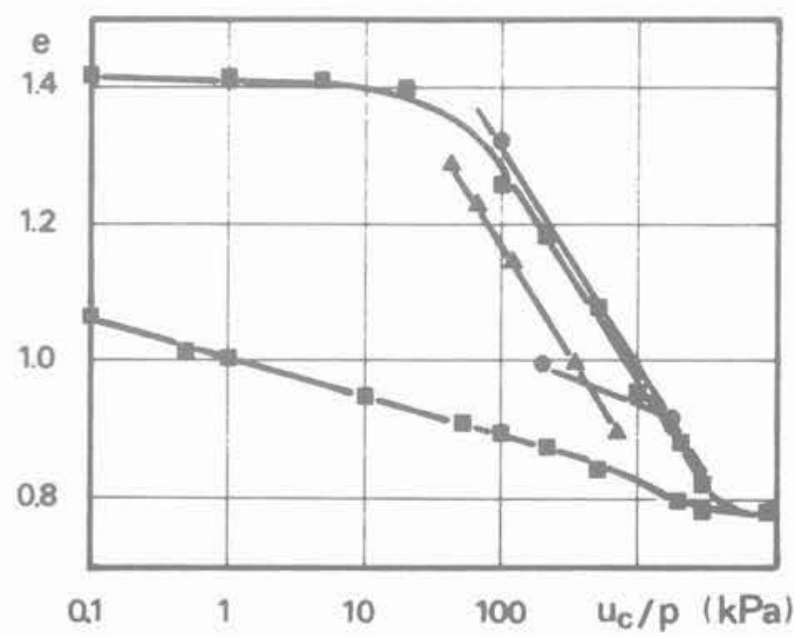

Fig. 1. - Comparaison des chemins de

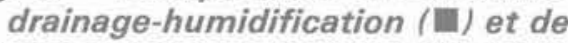
compression-décompression œdométrique (\) et isotrope $(\bullet)$ pour la kaolinite quasi saturée. Fig. 1. - Comparison between drying-wetting ( and oedometric ( $\mathbf{\Delta}$ ) or isotropic ( $\bullet$ compression-decompression paths for quasi-saturated kaolinite.

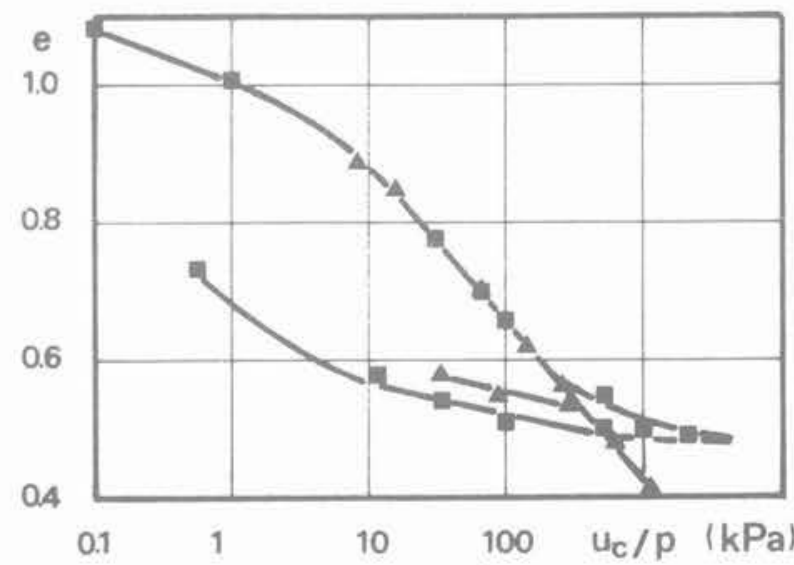

Fig. 2. - Comparaison des chemins de drainage-humidification (1) et de compression-décompression œdométrique $(\Delta)$ pour la marne quasi saturée.

Fig. 2. - Comparison between drying-wetting (ㄹ) and oedometric compression-decompression ( $\mathbf{\Delta}$ ) paths for quasi-saturated marl. 
Dans le cas des essais œdométriques, la contrainte $\sigma_{3}^{\prime}=\mathrm{K}_{0} \cdot \sigma_{1}^{\prime}$ a été calculée à partir de la valeur de $\mathrm{K}$ 。 mesurée pour la marne $\left(\mathrm{K}_{0}=0,3\right.$ pour le sol normalement consolidé : SOEPANDJI, 1986) et de la valeur déduite de la formule de JAKY $\left(K_{0}=0,5\right)$ pour la kaolinite. Pour les deux matériaux, l'état de départ de tous les essais était le même.

On constate que, dans le domaine saturé, les courbes de drainage-humidification ont la même allure que les courbes cedométriques et isotropes : au-delà d'une pression de "préconsolidation», ou limite élastique, ces courbes présentent une partie sensiblement linéaire, qui correspond à un comportement non réversible du matériau, sur laquelle on peut définir un indice de drainage. Dans le domaine élastique, on définira de même un indice d'humidification. Pour les deux matériaux, les droites sont parallèles et les indices correspondants sont égaux. Notons que, pour une valeur donnée de la contrainte, la courbe cedométrique se trouve à un indice des vides inférieur ou égal à celui de la courbe de drainage, tandis que celui-ci est légèrement plus élevé pour la compression isotrope. En conclusion, dans le domaine où les échantillons restent saturés, des incréments identiques de pression capillaire ou de contrainte mécanique produisent la même variation d'indice des vides. Par conséquent, si l'on reprend la notion de contrainte effective proposée par BISHOP, cela revient à admettre l'équivalence des effets de $\left(\sigma-u_{a}\right)$ et $u_{c}$ sur les variations de volume, c'est-à-dire à écrire : $x=1$ pour un sol quasi saturé $\left(\mathrm{S}_{\mathrm{r}} \approx 100 \%\right)$.

\subsection{Présentation des résultats des cycles complets de drainage-humidification}

Dans le cas général, l'application d'une pression capillaire à un échantillon de sol se traduira simultanément par une variation de son indice des vides et de son degré de saturation (ou encore de sa teneur en eau). C'est pourquoi il est nécessaire de disposer d'une représentation globale de l'état du matériau incluant tous ces paramètres, comme celle de la figure 3 qui présente les résultats d'un cycle de drainagehumidification sur la kaolinite. Cette figure comprend quatre graphiques qui se correspondent : le quadrant supérieur gauche (Indice des vides - Teneur en eau) montre les résultats d'une mesure de retrait par drainage. Sur ce graphique, la saturation de l'échantillon se traduit par une relation linéaire entre e et $w$ :

$$
\begin{gathered}
e=\left(\gamma_{s} / \gamma_{w}\right) \cdot w \\
\left(\gamma_{s} / \gamma_{w} ; \text { densité des grains solides }\right)
\end{gathered}
$$

représentée par une droite passant par l'origine du repère $(e-w)$. La limite de retrait $\left(w_{s}\right)$ peut être définie, à partir de cette courbe, à l'intersection de la droite de saturation et de la droite d'indice des vides constant (correspondant à une teneur en eau nulle). A titre de comparaison, les valeurs des limites de plasticité $\left(w_{p}\right)$ et de liquidité $\left(w_{1}\right)$ ont été reportées sur l'axe des teneurs en eau. Le quadrant supérieur droit (Indice des vides - pression capillaire ou $\mathrm{pF}$ ) est le plan œedométrique habituel dans lequel la contrainte verticale est remplacée par la pression capillaire, également en échelle logarithmique. Les deux autres quadrants font apparaî-

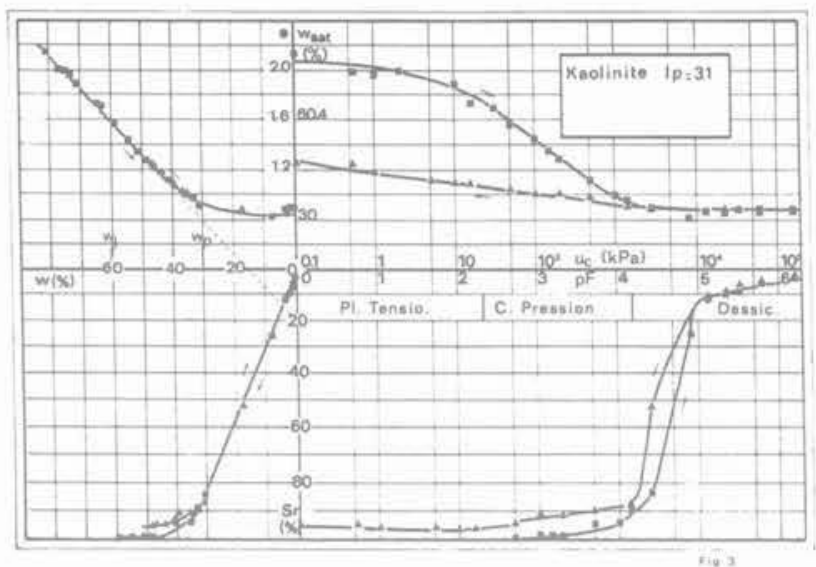

Fig. 3. - Représentation globale d'un cycle de drainage (E)-humidification ( $)$ sur la kaolinite $\left(w_{l}=1,5 w_{t}\right)$.

Fig. 3. - Global representation of a drying

(是)-wetting ( $\mathbf{\Delta})$ cycle on kaolinite $\left(w_{i}=1.5 w_{L}\right)$.

tre comme paramètre le degré de saturation $\mathrm{S}_{\text {f }}$, en relation avec la teneur en eau et la pression capillaire. La courbe degré de saturation - teneur en eau présente deux parties sensiblement linéaires correspondant d'une part à un degré de saturation proche de $100 \%$, d'autre part à une faible variation d'indice des vides du matériau. L'intersection entre les deux droites caractérise la teneur en eau pour laquelle il se produit une brusque diminution du degré de saturation, c'està-dire le point d'entrée d'air. Cette construction permet donc une détermination fiable et reproductible de ce point, par ailleurs très mal défini sur la courbe teneur en eau - indice des vides. A cette valeur de teneur en eau correspond une valeur de pression capillaire que l'on définira arbitrairement comme la "pression capillaire de désaturation " en drainage et de * saturation * en humidification.

La représentation constituée par les graphiques le $w)$ et $\left(S_{-}-w\right)$ est à rapprocher des abaques proposées par TERRACINA (ANDREI, 1977) et FREDLUND (1976) qui permettent de définir l'état du sol à partir d'une figure unique paramétrée par les différentes variables. Il apparaît néanmoins qu'une représentation dédoublée est plus claire et plus facile à utiliser qu'une représentation groupée.

\subsection{Influence de la nature des matériaux}

Les graphiques correspondant aux trois matériaux étudiés sont présentés sur les figures 3,4 et 5 . L'état initial des matériaux remaniés est une pâte saturée de teneur en eau égale à 1,2 ou 1,5 fois la limite de liquidité. A partir de cet état, les échantillons ont subi un drainage puis (sauf pour le limon) une humidification. Il faut d'abord noter la continuité des courbes de drainage et d'humidification, en dépit des différentes techniques expérimentales utilisées ( $\S 2.1$ ), ce qui conforte Thypothèse relative au rôle de la pression capillaire dans les changements de volume du sol. On remarquera par ailleurs l'absence d'hystérésis des courbes de 
retrait $e(w)$ et des courbes $S_{r}(w)$, alors que cette hystéresis est importante pour les courbes tracées en fonction de la pression capillaire. Cela signifie que, pour un état initial donné, la relation entre l'indice des vides (ou la densité sèche) et la teneur en eau est biunivoque et ne dépend pas du chemin de drainage-humidification suivi. Ce n'est quau niveau des pressions capillaires permettant d'accéder à un état donné que le chemin suivi joue un rôle. La seconde constatation est l'étendue du domaine de pression capillaire pour lequel le matériau reste quasi saturé. En effet, en drainage, il faut atteindre des pressions respectivement de $26 \mathrm{kPa}$ pour le limon, $500 \mathrm{kPa}$ pour la marne remaniée et $900 \mathrm{kPa}$ pour la kaolinite (points d'entrée d'air), pour noter un début de désaturation du sol. Dans les trois cas, les courbes se présentent de façon très semblable. mais avec des domaines de variation d'indices des vides et de teneurs en eau évidemment très différents : pour le limon, e varie de 0,8 à 0,6 lorsque la pression capillaire passe de $0,1 \mathrm{kPa}$ à $1000 \mathrm{MPa}$; pour la marne, la variation est de 1,1 à 0,4 et pour la kaolinite, de 2,1 à 0,9 .

Ces résultats peuvent être éclairés en considérant un modèle de sol composé de tubes capillaires rigides identiques, parallèles, placés verticalement. Si l'échantillon de sol est suffisamment haut par rapport à la remontée capillaire de l'eau résultant des interactions entre les différentes phases, solide, eau adsorbée et eau libre, air (caractérisées par la tension interfaciale entre fluides $\mathrm{A}$ et l'angle de contact statique $\theta$ ) et du rayon des capillaires $r$, le niveau de l'eau s'établira à une hauteur donnée par la loi de JURIN :

$$
\mathrm{H}_{\max }=2 \mathrm{~A} \cos \theta / \gamma_{w} \mathrm{r}
$$

$\left(\gamma_{w}\right.$ : poids volumique de l'eau

En revanche, si cette hauteur $\mathrm{H}_{\max }$ est supérieure à la hauteur $\mathrm{H}$ de l'échantillon, celui-ci sera saturé et l'équilibre sera obtenu par un ajustement de l'angle de contact, qui prendra une valeur $\alpha$ telle que :

$$
u_{c}=\gamma_{w} H=2 A \cos \alpha / r
$$

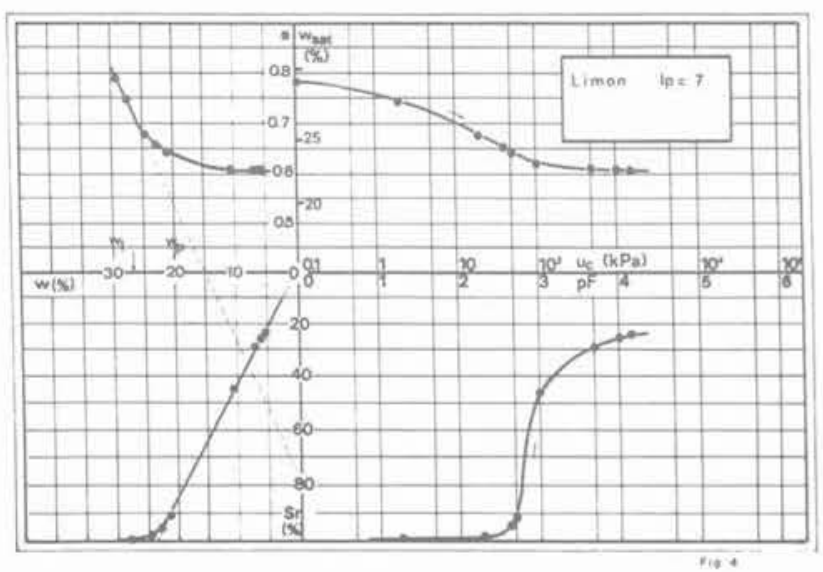

Fig. 4. - Représentation globale du chemin de drainage (৩) pour le limon de STERREBEEK $\left(w_{i}=1,2 w_{L}\right)$.

Fig. 4. - Global representation of a drying ( $\bullet$ ) path on STERREBEEK loam $\left(w_{i}=1.2 w_{L}\right)$.

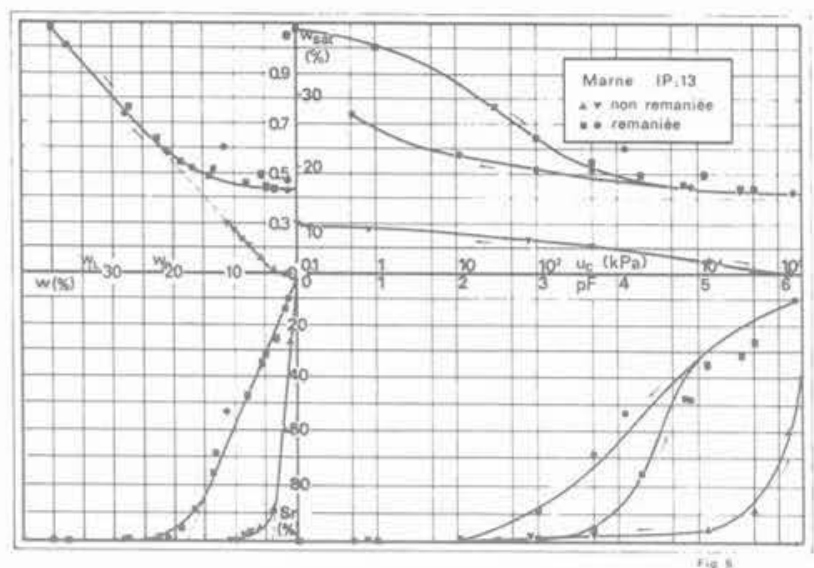

Fig. 5. - Représentation globale d'un cycle de drainage (茴)-humidification ( $)$ sur la marne remaniée $\left(w_{i}=1,2 w_{L}\right)$ et des chemins partiels de drainage ( $\mathbf{\Delta}$ ) et d'humidification ( $\nabla$ ) sur

la marne non remaniée $\left(w_{i}=6,2 \%\right)$.

Fig. 5. - Global representation of a drying

(E)-wetting (-) cycle on remolded marl $\left(w_{i}=1.2 w_{L}\right)$ and of a partial

drying ( $\mathbf{\Delta}$ )-wetting ( $\mathbf{\nabla})$ path on unremolded marl

$$
\left(w_{i}=6.2 \%\right) \text {. }
$$

Par conséquent, une augmentation de la pression capillaire ne se traduira pas immédiatement par une désaturation de l'échantillon, mais d'abord par une variation de l'angle de contact, de $\alpha$ à $\theta$, avant que l'eau ne se mette en mouvement. En fait, cet angle d'équilibre statique $\theta$ est compris entre deux valeurs $\theta_{\text {min }}$ et $\theta_{\text {max }}$ correspondant à un déplacement relatif des phases l'une par rapport à l'autre. En drainage, la mise en mouvement de l'eau aura lieu lorsque la pression capillaire sera supérieure à la valeur :

$$
\left(\mathrm{u}_{c}\right)_{\max }=2 \mathrm{~A} \cos \theta_{\min } / \mathrm{r}
$$

En humidification, le déplacement de l'eau sera caractérisé par :

$$
\left(\mathrm{u}_{\mathrm{e}}\right)_{\min }=2 \mathrm{~A} \cos \theta_{\max } / \mathrm{r}
$$

On retrouve donc bien, à partir de ce modèle élémentaire, d'une part l'existence d'un domaine de pressions capillaires dans lequel l'échantillon reste saturé, domaine qui dépend notamment des interactions solide-liquide et de la taille des pores, d'autre part l'écart entre les pressions capillaires de désaturation et de saturation (Hystérésis).

\subsection{Influence du remaniement sur le comportement de la marne}

Des essais de drainage et d'humidification ont également été effectués sur les échantillons de marne non remaniée, à partir de la teneur en eau naturelle $(6,2 \%)$. Les résultats de ces essais ont été représentés sur la figure 5 , en même temps que les résultats des essais sur échantillons remaniés à $w_{1}=40 \%$.

Dans tout le domaine de pressions capillaires étudié, l'indice des vides des échantillons non remaniés reste 
inférieur à l'indice des vides final des échantillons remaniés obtenu pour $u_{c}=1000 \mathrm{MPa}$. D'autre part, la variation totale d'indice des vides des échantillons non remaniés $(\delta e=0,22)$ est très faible devant celle des échantillons remaniés $(\delta e=0,56)$. Cette différence de comportement peut être attribuée à la forte cimentation de la marne in situ et à son état initial très compact (SOEPANDJI, 1986).

La comparaison entre les courbes de retrait $(e-w)$ des matériaux remanié et non remanié montre très clairement que la limite de retrait dépend du remaniement : la valeur est de $17 \%$ pour la marne remaniée tandis qu'elle n'est que de $4 \%$ pour le sol naturel. On retrouve sur ce graphique l'alignement des points expérimentaux sur la même droite de saturation, caractérisée par la densité des grains solides. On constate enfin que les échantillons non remaniés restent quasi saturés jusqu'à des valeurs de pression capillaire très élevées, de l'ordre de $30 \mathrm{MPa}$ - selon la procédure de détermination indiquée précédemment - au lieu de $400 \mathrm{kPa}$ pour la marne remaniée. Ceci peut s'expliquer par le fait que la cimentation et la surconsolidation ont réduit la taille des pores de façon considérable, entraînant une augmentation de la pression capillaire de désaturation (cf. § 3.3.).

\subsection{Influence de l'état initial et du chemin suivi}

Comme il a été indiqué plus haut, les échantillons de kaolinite, préparés à partir d'une pâte à teneur en eau initiale égale à 1,5 fois la limite de liquidité, ont été soumis à des préconsolidations initiales différentes, par des contraintes mécaniques ou capillaires, ou par une combinaison de celles-ci (figure 6).

Les résultats obtenus lors du drainage sont représentés sur la figure 7 : la courbe supérieure, dans le repère $\left(e-u_{c}\right)$ correspond aux échantillons n'ayant subi aucune préconsolidation (série K1 : pâte); la courbe intermédiaire correspond aux échantillons soumis à une préconsolidation œedométrique à $\sigma^{\prime}=28 \mathrm{kPa}$ (série K2), tandis que la courbe inférieure est relative à une contrainte œdométrique $\sigma^{\prime}=55 \mathrm{kPa}$ (série K3). On note tout d'abord que l'indice des vides correspondant à une pression capillaire donnée est d'autant plus faible que la contrainte de préconsolidation est plus élevée. Ceci se traduit sur les courbes de retrait, par une limite de retrait plus faible pour les échantillons les plus fortement préconsolidés, en relation avec des indices des vides finaux (pour $u_{f}=1000 \mathrm{MPa}$ ) plus faibles. La limite de retrait varie de $33 \%$ pour la pâte à $28 \%$ pour les échantillons consolidés sous $55 \mathrm{kPa}$. Par conséquent, comme cela a été indiqué dans le cas des échantillons non remaniés, la limite de retrait dépend de l'état initial et n'est pas une caractéristique intrinsèque du matériau. Parallèlement, on observe une augmentation de la pression capillaire de désaturation avec la préconsolidation initiale des échantillons. Celle-ci peut résulter de la réduction de la taille des pores lors de la consolidation.

Par ailleurs, l'indice de drainage, pente de la partie linéaire de la courbe de drainage $\left(e-u_{c}\right)$ est identi-

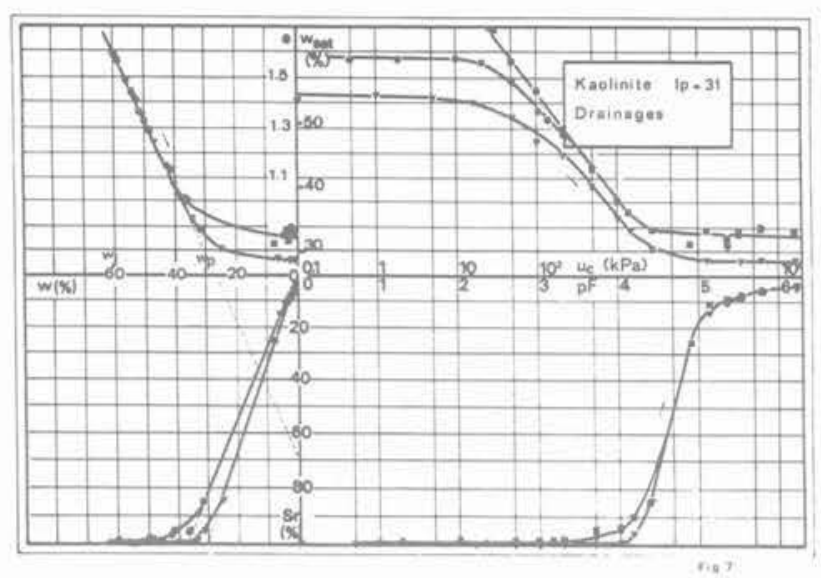

Fig. 7. - Influence de la préconsolidation initiale des échantillons de kaolinite sur les chemins de drainage (10 : Pate;

- : Consolid. $28 \mathrm{kPa} ; \nabla$ : Consolid. $55 \mathrm{kPa}$ ).

Fig. 7. - Influence of the initial preconsolidation stress of kaolinite samples on the drying paths

(1) slurry; : Consolid. $28 \mathrm{kPa}$; $\mathbf{\nabla}$ : Consolid. $55 \mathrm{kPa}$ ),

que, dans tous les cas, à celui de la courbe de drainage vierge sur la pâte.

Sur le chemin d'humidification, l'état initial des échantillons est obtenu par application d'une contrainte mécanique ou capillaire à la pâte. Pour les échantillons K4 et K5, les pressions capillaires imposées sont respectivement de $1500 \mathrm{kPa}$ et $1000 \mathrm{MPa}$. Les échantillons K6 ont été soumis successivement à une contrainte œedométrique de $55 \mathrm{kPa}$ et à une dessication totale jusqu'à $u_{f}=1000 \mathrm{MPa}$. Dans tous les cas, l'humidification est poursuivie jusqu'à $u_{c}=0,1 \mathrm{kPa}$. Les résultats sont indiqués sur la figure 8.

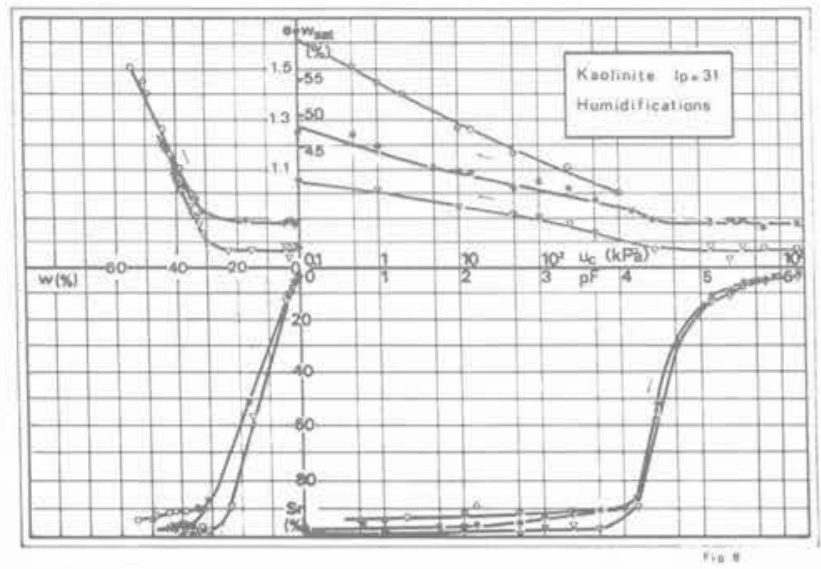

Fig. 8. - Influence de la préconsolidation et du drainage initiaux des échantillons de kaolinite sur les chemins d'humidification $10:\left(u_{c}\right)_{i}=1500 \mathrm{kPa} ;{ }^{*}:\left(u_{c_{i}}\right)_{i}=1000 \mathrm{MPa}$; $\nabla:$ Consolid. $55 \mathrm{kPa} /\left(u_{l_{i}}=1000 \mathrm{MPa}\right)$.

Fig. 8. - Influence of the initial (mechanical and capillary) preconsolidation stress of kaolinite samples on the wetting paths

$10:\left(u_{c}\right)_{i}=1500 \mathrm{kPa} ; *\left(u_{c}\right)_{i}=1000 \mathrm{MPa}$; $\nabla:$ Consolid. $55 \mathrm{kPa}$ and $\left.\left(u_{c}\right)_{i}=1000 \mathrm{MPa}\right)$. 
Il apparait tout d'abord que, dans le domaine partiellement saturé, l'indice des vides ne varie pratiquement pas et que l'humidification ne provoque pas de gonflement. En revanche, pour les teneurs en eau supérieures à la limite de retrait, il se produit une variation d'indice des vides sensiblement linéaire avec le logarithme de la pression capillaire. La pente de cette droite, ou indice d'humidification, apparaît d'autant plus importante que la préconsolidation du sol est plus faible. Enfin, l'indice des vides final, obtenu pour $\mathrm{u}_{c}=$ $0,1 \mathrm{kPa}$, est nettement inférieur à l'indice des vides initial des échantillons des séries $\mathrm{K} 1$ et $\mathrm{K} 3$ ce qui confirme le fait que le premier cycle d'hystérésis n'est pas fermé.

\section{CONCLUSION}

Les essais qui ont été présentés dans cet article avaient d'abord pour but de montrer l'intérêt d'une représentation globale de l'état du matériau au cours d'un cycle de drainage-humidification. Cette représentation permet notamment de suivre l'évolution de la saturation du sol, de relier les caractéristiques de retrait aux caractéristiques de drainage et d'humidification, de mettre en évidence les erreurs éventuelles de mesure, etc. Par ailleurs, le rôle de l'état initial du matériau a été précisé, que cet état résulte d'un drainage ou d'une compression mécanique préalables, ou encore du remaniement. Il a été montré, en particulier, que les courbes de drainage et d'humidification sont extrêmement sensibles au chemin suivi lors de la préparation de l'échantillon, de même que la limite de retrait.

Enfin, la comparaison entre les essais de drainagehumidification et les essais mécaniques de compression-décompression cedométriques et isotropes a montré qu'il existe, dans les conditions d'essai considérées, une équivalence entre la contrainte mécanique appliquée et la pression capillaire tant que le sol reste saturé. Ainsi, pour un sol saturé, les variations de volume ne dépendent que des variations de la contrainte effective :

$$
\sigma^{\prime}=\sigma-\mathbf{u}_{w},
$$

que celles-ci proviennent des variations de $\sigma$ ou de $u_{w}$.

\section{Remerciements}

Les auteurs remercient M. D. TESSIER, de IIIN.R.A. (Versailles), pour l'aide qu'il a apportée à la mise au point des dispositifs de mesure de pression capillaire, ainsi que M. le Professeur J.C. VERBRUGGE (Faculté des Sc. Agronom. de Gembloux) qui a fourni le limon de Sterrebeek.

\section{RÉFÉRENCES}

ANDREI S. (1977), Propriétés hydrauliques et mécaniques des sols non saturés. Revue Française de Géotechnique, $n^{\circ} 2$, pp. 51-78.

BARDEN L., MADEDOR A.O., SIDES G.R. (1969), Volume change characteristics of unsaturated clays. Journal of the Soil Mechanics and Found. Div., Pro- ceedings of the A.S.C.E., vol. $95, n^{\circ}$ S.M.1., janvier 1969, pp. 33-51.

BISHOP A.W. et BLIGHT G.E. (1963), Some aspects of effective stress in saturated and unsaturated soils. Géotechnique, vol. 13, pp. 177-197.

BLIGHT G.E. (1966), Strength characteristics of dessicated clays. Journal of the Soil Mechanics and Foundation Division, Proceedings of the A.S.C.E., vol. 92, n S.M.6, décembre 1966, pp. 19-37.

BLIGHT G.E. (1967), Effective stress evaluation for unsaturated soils. Journal of the Soil Mechanics and Foundation Division, Proceedings of the A.S.C.E., vol. 93, n० S.M.2, mars 1967, pp. 125-148.

COLEMAN J.D. et MARSH A.D. (1961), An investigation of the pressure-membrane method for measuring the suction properties of soil. Soil Science, vol. 12, pp. 343-362.

CRONEY D. et COLEMAN J.D. (1960), Pore pressure and suction in soil. Conference on Pore Pressure and Suction in soil (British National Soc, of Soil Mechanics), Butterworth, London, pp. 31-37.

DANIEL D.E. (1983), Permeability test for unsaturated soil. Geotechnical Testing Journal, vol. $6, n^{\circ} 2$, juin 1983, pp. 81-86.

FREDLUNG D.G. et MORGENSTERN N.R. (1976), Constitutive relations for volume changes in unsaturated soils. Canadian Geotechnical Journal, vol. 13, $\mathrm{n}^{\circ} 3$, août 1976, pp. 261-275.

FREDLUND D.G. et MORGENSTERN N.R. (1977), Stress state variables for unsaturated soils. Journal of the Geotechnical Division. Proceedings of the A.S.C.E., vol, 103, G.T. 5, pp. 447-465.

GULHATI S.K. et SATIJA B.S. (1981), Shear strength of partially saturated soils. Proceedings of the 10th International Conference on Soil Mechanics and Foundation Engineering, Stockholm, pp. 609-612.

HO. D.Y.F, et FREDLUND D.G. (1982), Increase in strength due to suction for two Hong Kong soils. Proceedings of the Conference on Engineering and Construction in Tropical and Residual Soils, A.S.C.E., Honolulu, janvier 1982, pp. 263-295.

JENNINGS J.E.B. (1961), A revised effective stress law for use in the prediction of the behaviour of unsaturated soils. Conference on Pore Pressure and Suction in Soils (British National Society of Soil Mechanics) Butterworth, London, pp. 26-30.

KASSIF G. et BENSHALOM A. (1971), Experimental relationship between swell pressure and suction. Géotechnique, vol. $21, n^{\circ} 3$, pp. 245-255.

LLORET A. et ALONSO E.E. (1985), State surfaces for partially saturated soils. Proceedings of the 11th International Conference on Soil Mechanics and Foundation Engineering, San Francisco.

MATYAS S.E. et RADHAKRISHNA H.S. (1968), Volume change characteristics of partially saturated soils. Géotechnique, vol. $18, n^{\circ} 4$, pp. $432-448$. 
PADDAY J.F. (1969), Theory of surface tension in Surface and Colloid Science, édité par E. MATIJEVIC, Wiley Interscience, New York.

SOEPANDJI B. (1986), Contribution à l'étude des propriétés mécaniques des marnes et argiles soumises à de fortes pressions. Thèse présentée à l'École Centrale de Paris, soutenue le 27 juin 1986 , pour obtenir le grade de Docteur.

TESSIER D. et BERRIER J. (1979), Utilisation de la microscopie électronique à balayage dans l'étude des sols, Science du Sol, bulletin de l'A.F.E.S., $n^{\circ} 1$, pp. 67-82.

TESSIER D. (1975), Recherches expérimentales sur l'organisation des particules dans les argiles. Thèse présentée au Conservatoire national des Arts et Métiers, soutenue le 25 juin 1975, pour obtenir le Diplôme d'ingénieur C.N.A.M.

VERBRUGGE J.C. (1974), Contribution à la mesure de la succion et de la pression interstitielle dans les sols non saturés. Thèse présentée à l'Université libre de Bruxelles, soutenue en juin 1974 pour obtenir le grade de Docteur.

VERBRUGGE J.C. (1979), Étude des sols non saturés au moyen d'un appareil triaxial équipé d'un psychromètre à effet Peltier. Proceedings of the 7 th European Conference on soil Mechanics and Foundation Engin., Brighton-London, vol. 2, pp. 161-164.

WIGNYODARSONO S.L. (1985), Étude de l'action des surfactants sur les propriétés hydrauliques et mécaniques des argiles. Thèse présentée à l'École Centrale de Paris, soutenue le 2 juillet 1985 pour obtenir le grade de Docteur-Ingénieur.

ZERHOUNI M.I. (1985), Les sols non saturés : étude et méthodes d'investigation. Mémoire de DEA présenté à l'École centrale de Paris, soutenu le 26 juin 1985. 